\title{
Molecular characteristics of homologous salivary adenoid cystic carcinoma cell lines with different lung metastasis ability
}

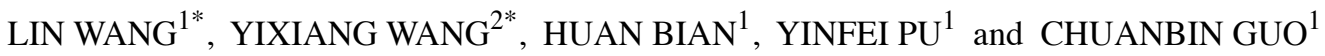 \\ ${ }^{1}$ Department of Oral and Maxillofacial Surgery; ${ }^{2}$ Central Laboratory, Peking University \\ School and Hospital of Stomatology, Beijing, P.R. China
}

Received January 16, 2013; Accepted April 15, 2013

DOI: $10.3892 /$ or.2013.2460

\begin{abstract}
Although the homologous salivary adenoid cystic carcinoma (SACC) cell lines SACC-83 and SACC-LM have already been used as SACC models to investigate the underlying mechanisms of metastasis, the molecular features of these SACC cell lines remain unclear. We screened 136 genes related to metastasis in order to investigate the biological and molecular properties of these two cell lines by short tandem repeat (STR) profiling, immunostaining, transwell invasion assay, real-time PCR and western blotting. STR and immunostaining results showed that SACC-83 and SACC-LM are homologous cancer cell lines, derived from adenoepithelial cells and, to date, are not contaminated by each other or other cancer cell lines. Transwell invasion assay results showed that SACC-LM had increased invasion ability compared to SACC-83. 29 of the 136 differentially expressed genes including EREG, S100P, cyclooxygenase (COX)-2, phospho-Akt (p-Akt), matrix metalloproteinase (MMP)-1, MMP-2, MMP-3, MMP-9, MMP-13 and MMP-14 were found following gene screening in SACC-83 and SACC-LM cells. Compared with SACC-83, SACC-LM presents higher expression of COX-2, S100P and lower expression of MMP-2, p-Akt, which could be candidates for identifying the homologous pair cell lines.
\end{abstract}

\section{Introduction}

Cell biology is an important field of study and a valuable tool used to investigate cell proliferation, differentiation,

Correspondence to: Dr Yixiang Wang, Central Laboratory, Peking University School and Hospital of Stomatology, 22 Zhongguancun Avenue South, Haidian District, Beijing 100081, P.R. China

E-mail: kqwangyx@bjmu.edu.cn

Dr Chuanbin Guo, Department of Oral and Maxillofacial Surgery, Peking University School and Hospital of Stomatology, 22 Zhongguancun Avenue South, Haidian District, Beijing 100081, P.R. China

E-mail:guodazuo@sina.com

*Contributed equally

Key words: salivary adenoid cystic carcinoma cell line, molecular characteristics, short tandem repeat development, aging, apoptosis or death, carcinogenesis and metastasis (1). Researchers have established thousands of cell lines as models to explore the corresponding tissues. However, with the increasing number of immortalized cell lines established, cross-contamination of cell lines is also becoming a more frequently encountered problem $(2,3)$. Cell line cross-contamination may alter the characteristics of the cultures, leading to inaccurate results and affecting the credit score of the research group $(3,4)$. Therefore, the identification of immortalized cell lines is key for cell biology, particularly for cancer cell biology, as most immortalized cell lines are cancer cell lines.

Cell lines derived from different individuals are generally easier to be identified as they normally have different short tandem repeat (STR) profiling $(5,6)$. However, for the cell lines derived from the same individual with different biological properties, it is unclear whether they have contaminated each other as they have the same STR loci. Thus, it is necessary to identify the molecular markers as well as the distinct biological characteristics in certain aspects to determine the homologous cancer cell lines.

In the present study, we introduced the comparable salivary adenoid cystic carcinoma (SACC) cell lines, the parental cell line SACC-83, with a low lung metastasis rate, and its daughter cell line SACC-LM, with a high lung metastasis rate $(7,8)$. SACC-83 was isolated from a patient pathologically diagnosed in 1983 with SACC in the sublingual gland, and SACC-LM was generated from lung metastatic lesions through 5-round selections starting from intravenous injection of SACC-83 cells (8).

Cell cross-contamination is becoming an increasingly serious issue $(3,4)$ and although the homologous SACC cell lines SACC-83 and SACC-LM have recently been used as a SACC model to investigate the underlying mechanism of metastasis (8-12), the molecular features of these SACC cell lines remain unclear. In this study, we screened the expression pattern of 136 genes in these two cell lines in order to find the molecular properties of the two SACC cells and to avoid the misuse of the two cell lines in the future.

\section{Materials and methods}

Cell culture. Human SACC cell lines, SACC-83 and SACC-LM, established in our laboratory, were cultured in RPMI-1640 (Gibco, Grand Island, NY, USA) supplemented 
with $10 \%$ fetal bovine serum (Gibco), $100 \mathrm{U} / \mathrm{ml}$ penicillin and $100 \mu \mathrm{g} / \mathrm{ml}$ streptomycin in a humidified incubator at $37^{\circ} \mathrm{C}$ with $5 \% \mathrm{CO}_{2}$.

STR analysis. DNA was extracted from SACC-83 and SACC-LM cells as previously described (10). STR profiling PCR was carried out in the ABI 3100 genetic analyzer machine by the use of $10 \mathrm{ng}$ of DNA as a template and the Goldeneye ${ }^{\mathrm{TM}} 16 \mathrm{C}$ STR detection kit.

Immunostaining. SACC-83 and SACC-LM cells were plated into 24-well plates containing round coverslides in RPMI-1640 culture medium for $24 \mathrm{~h}$, then fixed with $4 \%$ paraformaldehyde for $10 \mathrm{~min}$, and subjected to immunostaining assay to identify the cell type of SACC-83 and SACC-LM. Briefly, the glass coverlids were rinsed twice with PBS, and endogenous peroxidase was blocked by the use of $3 \%$ hydrogen peroxide in PBS for $10 \mathrm{~min}$. The samples were blocked with normal goat serum for $1 \mathrm{~h}$, incubated with anti-human pan-cytokeratin (pan-CK), cytokeratin (AE1), CK8/18 and S100P (Zhongshan Golden Bridge Biotechnology Co., Ltd., Beijing, China) overnight at $4^{\circ} \mathrm{C}$, followed by horseradish peroxidase-conjugated immunoglobulin for $30 \mathrm{~min}$, developed for color with peroxidase substrate 3,3'-diaminobenzidine (DAB), counterstained with hematoxylin, and recorded using an Olympus DP controller (Olympus, Tokyo, Japan).

Transwell invasion assay. Cell invasion assays were performed using transwell chambers with a polycarbonate membrane (Millipore, Bedford, MA, USA) coated with $40 \mu \mathrm{l}$ diluted matrix gel (BD Biosciences). Cells were trypsinized and seeded at $1 \times 10^{5}$ cells/well/ $0.1 \mathrm{ml}$ serum-free RPMI-1640 medium in the upper chambers, and $0.5 \mathrm{ml}$ of RPMI-1640 medium supplemented with $20 \%$ FBS was added into each lower chamber. $48 \mathrm{~h}$ after incubation, cells on the surface of the membrane were wiped off, and the membranes were fixed with $95 \%$ ethanol and stained with $1 \%$ crystal violet (Sigma-Aldrich). The invaded cells clinging to the bottom of the membrane were evaluated by light microscopy at 10x magnification, and by measurement of optical density $(\mathrm{OD})_{570}$ for the solution derived from the crystal violet discolored from the invaded cells by using $300 \mu \mathrm{l}$ of $33 \%$ glacial acetic acid. All experiments were performed in triplicate and similar results were obtained from three independent experiments.

Quantitative PCR ( $q P C R$ ) analysis. Total RNA was extracted from SACC-83 or SACC-LM cell lines using the TRIzol reagent according to the manufacturer's instructions (Invitrogen, Carlsbad, CA, USA). Complementary DNA was reverse transcribed by the use of $2.5 \mu \mathrm{g}$ of RNA as a template. $\mathrm{qPCR}$ was performed using the ABI 7500 real-time PCR machine coupled with SYBR Green chemistry (Applied Biosystems, Foster City, CA, USA). All PCR reactions were in $20 \mu \mathrm{l}$ of total volume containing $10 \mu 1$ of SYBR Green PCR master mix, $50 \mathrm{ng}$ cDNA, $250 \mathrm{nM}$ of each primer. All amplifications were performed in triplicate for each sample and repeated three times. The thermal cycling was $10 \mathrm{~min}$ at $95^{\circ} \mathrm{C}$, followed by 40 cycles at $95^{\circ} \mathrm{C}$ for $15 \mathrm{sec}$, at $60^{\circ} \mathrm{C}$ for $60 \mathrm{sec}$. The specificity of amplification was monitored using the dissociation curve of the amplified product. Relative expression of the target genes was calculated using the $2^{-\Delta \Delta \mathrm{Ct}}$ method.

Western blotting. Cells were harvested and lysed in RIPA buffer with protease inhibitors (Applygen Technologies, Inc., Beijing, China). Protein concentration was determined using the BCA Protein Assay (Thermo Fisher Scientific, Waltham, MA, USA), and $40 \mu \mathrm{g}$ of protein was loaded for each sample. Proteins were separated on a sodium dodecyl sulfate polyacrylamide gel electrophoresis (SDS-PAGE) and transferred to a polyvinylidene difluoride membrane. The membranes were blocked in 5\% non-fat dry milk for $1 \mathrm{~h}$ and probed with antibodies against S100P (Sino Biological Inc., Beijing, China), S100A4 (BD Biosciences), matrix metalloproteinase (MMP)-1, MMP-2, MMP-9, MMP-12, MMP-14,MMP-17 (Epitomics Inc., Burlingame, CA, USA), cyclooxygenase (COX)-2, phosphoAkt (p-Akt) (Cell Signaling Technology, Danvers, MA, USA), and $\beta$-actin (Santa Cruz Biotechnology, Inc., Santa Cruz, CA, USA) separately at $4^{\circ} \mathrm{C}$ overnight. Following incubation with peroxidase-linked secondary antibodies, immunoreactive proteins were visualized by enhanced chemiluminescence (ECL) reagent (Applygen Technology, Inc.).

Statistical analysis. Quantitative data are expressed as the means \pm SD and compared using a t-test through SPSS software, version 13.0 (SPSS, Inc., Chicago, IL, USA). P $<0.05$ was considered to indicate a statistically significant difference.

\section{Results}

STR profiling analysis. To investigate whether SACC- 83 and SACC-LM cell lines originated from the same patient, STR assay was performed using Goldeneye ${ }^{\mathrm{TM}} 16 \mathrm{C}$ STR detection kit. The results are shown in Fig. 1. SACC-83 and SACC-LM had the identical STR profiling in 16 gene foci [D19S433 $(13,13)$; D5S818 (11, 12); D21S11 (27, 30); D18S51 (13, 16); PentaD $(9,12)$; D3S1358 (15, 16, 18); D13S317 (10, 12, 13.3); D2S1338 $(17,23)$, D7S820 (10, 12), D16S539 (9, 10), CSF1PO (10, 11); vWA (14, 16); D8S1179 (12, 16, 17); D12S391 (20, 21); FGA $(18,21)$ and amelogenin $(X, X)]$. Compared with HeLa cell STR profiling [D19S433 (13, 14); D5S818 (11, 12); D21S11 (27, 28); D18S51 (16, 16); PentaD (8, 15); D3S1358 (15, 18); D13S317 (12, 14); D2S1338 (17, 17), D7S820 (8, 12), D16S539 $(9,10)$, CSF1PO (9, 10); vWA $(16,18)$; D8S1179 $(12,13)$; D12S391 $(20,25)$; FGA $(21,21)$ and amelogenin $(X, X)]$ only 3 of the 16 STR foci (D5S818, D16S539 and amelogenin) were the same as those in SACC-83 and SACC-LM. The remaining 13 STR foci listed above were different from SACC-83 and SACC-LM. The results indicated that the SACC-83 and SACC-LM cell lines are derived from the same patient and, most importantly, they have thus far not been contaminated by HeLa cells (Fig. 1).

SACC-83 and SACC-LM derive from adenoepithelial cells. To verify the cell type of SACC-83 and SACC-LM, immunostaining was performed by using the epithelial markers pan-cytokeratin and cytokeratin AE1, and the luminal markers CK8/18 and S100P. Immunostaining results showed that both SACC-83 and SACC-LM cells were positive not only for epithelial, but also for luminal markers (Fig. 2). This indicated that both SACC-83 and SACC-LM originated from oral adenoepithelial cells. However, 

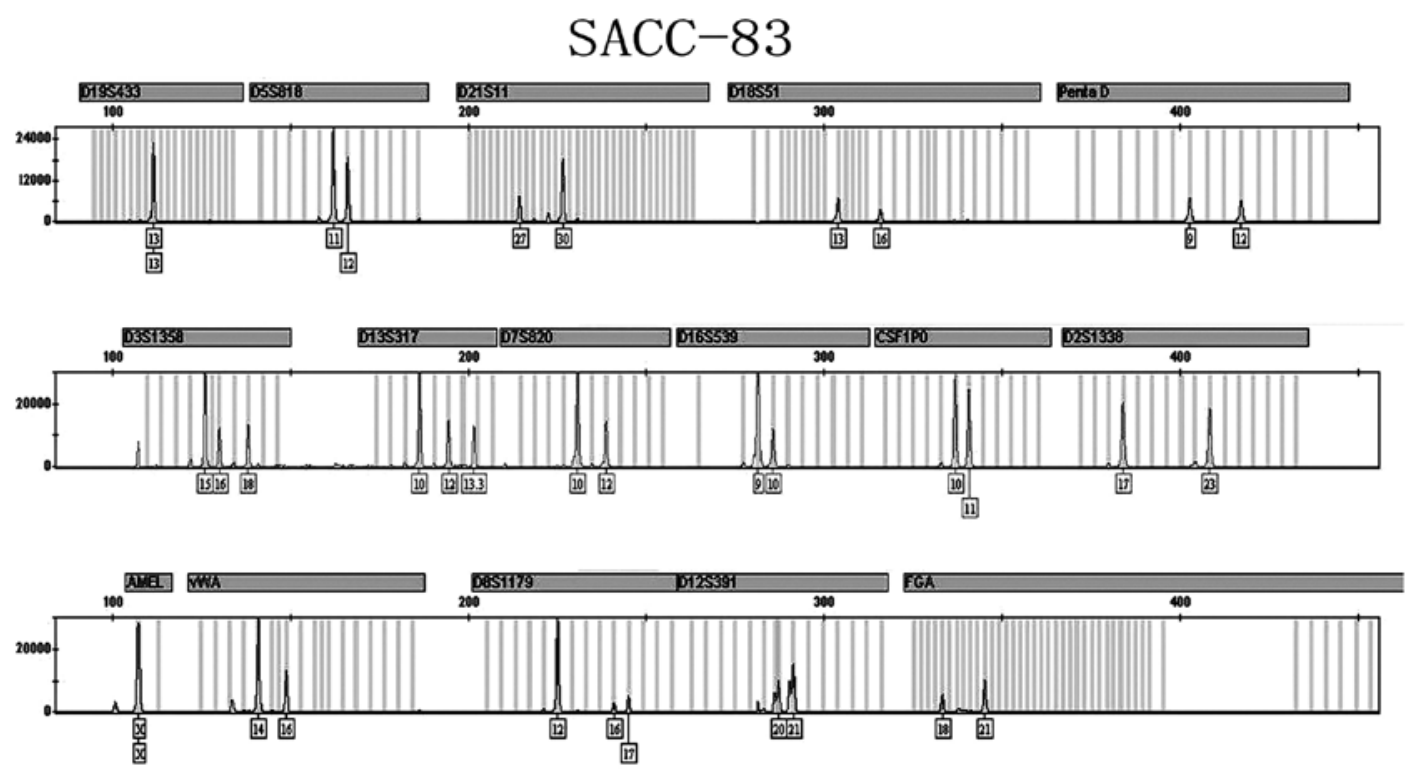

SACC-LM
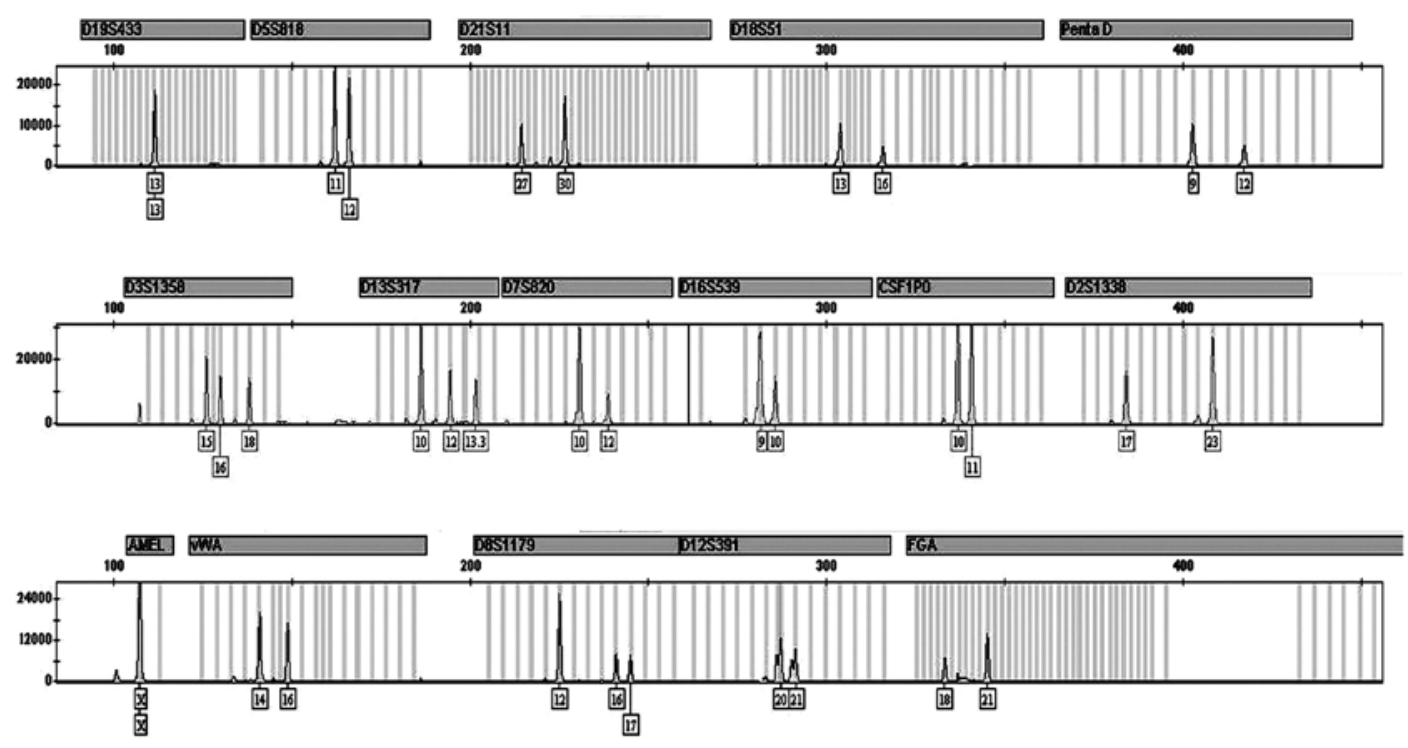

Figure 1. Short tandem repeat (STR) analysis. SACC-83 and SACC-LM cells have identical STR profiling, which indicates SACC-83 and SACC-LM derived from the same patient and were not contaminated by other cancer cell lines.

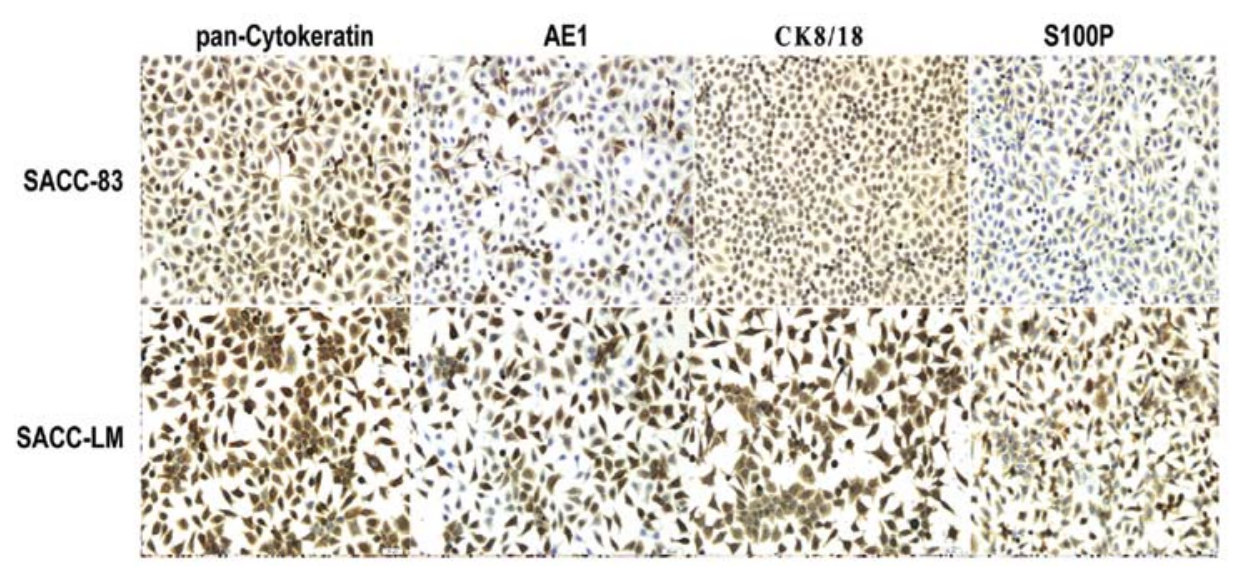

Figure 2. Identification of SACC-83 and SACC-LM cell type through immunostaining using the epithelial markers pan-cytokeratin and cytokeratin AE1, and the luminal markers CK8/18 and S100P. SACC-83 and SACC-LM were positive for the four markers, indicating that SACC-83 and SACC-LM originated from oral adenoepithelial cells. S100P was highly expressed in SACC-LM compared with SACC-83, which indicates that SACC-83 and SACC-LM have different molecular features as well as biological properties. 

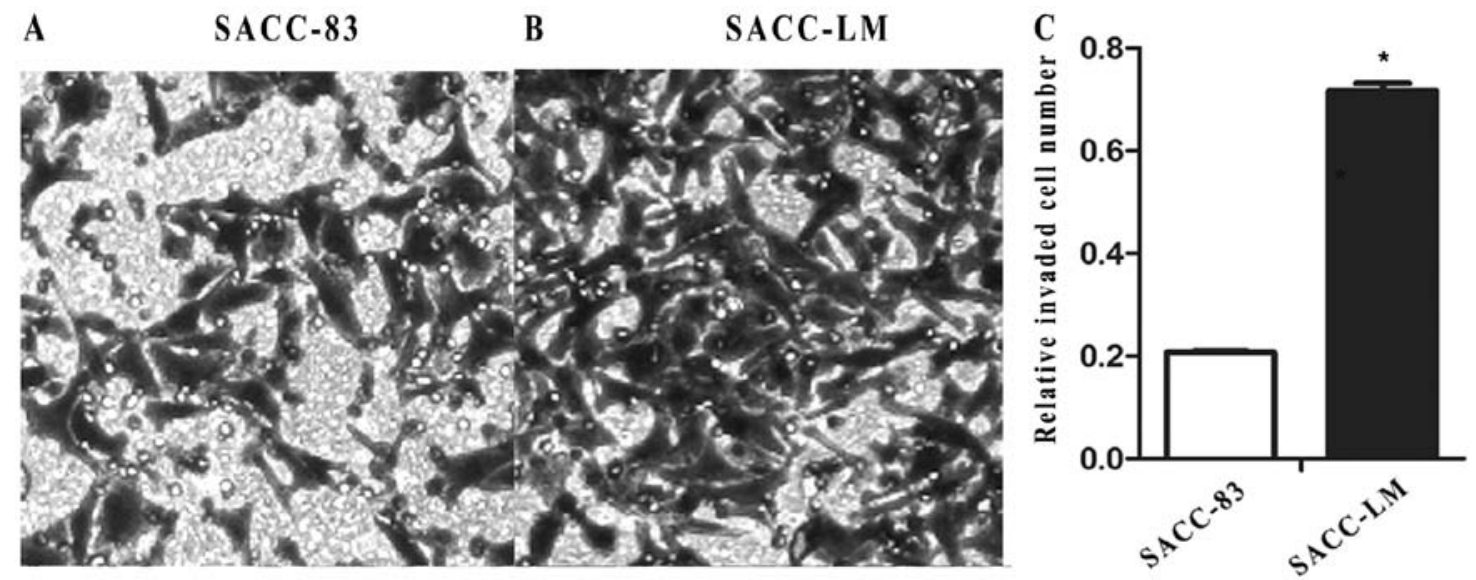

Figure 3. (A and B) Transwell invasion assay. The invaded SACC- 83 and SACC-LM cells are shown on the bottom surface of the membrane $48 \mathrm{~h}$ postinoculation. (C) The histogram shows the relative invaded SACC-83 and SACC-LM cell numbers by measuring $\mathrm{OD}_{570}$ for the solution derived from the crystal violet discolored from the invaded cells. ${ }^{*} \mathrm{P}<0.01$.

Table I. The list of the 29 differentially expressed genes in SACC-LM compared to SACC-83.

\begin{tabular}{|c|c|c|c|}
\hline Abbreviation & $\begin{array}{c}\text { GenBank } \\
\text { accession no. }\end{array}$ & Fold-change & P-value \\
\hline
\end{tabular}

\begin{tabular}{llrl} 
Increased & & & \\
COX-2 & NM_000963 & 6.9 & 0.001 \\
MMP-3 & NM_002422 & 2.1 & 0.001 \\
MMP-9 & NM_004994 & 2.3 & 0.001 \\
MMP-14 & NM_004995 & 2.9 & 0.002 \\
BMP7 & NM_001719 & 66.8 & 0.032 \\
S100P & NM_005980 & 8.4 & 0.001 \\
S100A2 & NM_005978 & 2.7 & 0.001 \\
S100A13 & NM_001024210 & 1.6 & 0.039 \\
S100A16 & NM_080388 & 2.1 & 0.047 \\
KRT15 & NM_002275 & 8.4 & 0.031 \\
EREG & NM_001432 & 9.8 & 0.002 \\
WNT2 & NM_003391 & 2.3 & 0.04 \\
WNT3 & NM_030753 & 1.8 & 0.005 \\
Decreased & & & \\
MMP-1 & NM_001145938 & -2.4 & 0.006 \\
MMP-2 & NM_001127891 & -3.2 & 0.006 \\
uPA & NM_001145031 & -6.9 & 0.033 \\
Trail & NM_001190942 & -2.6 & 0.046 \\
FXYD5 & NM_001164605 & -1.5 & 0.031 \\
KRT7 & NM_005556 & -2.7 & 0.019 \\
KRT16 & NM_005557 & -2.2 & 0.012 \\
MCAM & NM_006500 & -2.5 & 0.008 \\
NME4 & NM_005009 & -2.3 & 0.035 \\
NOTCH1 & NM_017617 & -2.4 & 0.036 \\
CCL3 & NM_002983 & -2.4 & 0.006 \\
CCL15 & NM_032965 & -3.4 & 0.049 \\
CCL19 & NM_006274 & -2.6 & 0.036 \\
CXCL5 & NM_002994 & -2.7 & 0.045 \\
BMP3 & NM_001201 & -2.4 & 0.039 \\
BMP5 & NM_021073 & -2.3 & 0.022 \\
\hline & & & \\
\hline
\end{tabular}

S100P expression in SACC-LM was higher than that in SACC83, which indicated SACC-LM had different molecular features compared with SACC-83.

Invasion ability of SACC-83 and SACC-LM. Prior to the investigation of the molecular differences between SACC-83 and SACC-LM, the invasion ability was explored based on the results in the previous studies. The transwell assay showed that the invaded SACC-83 cells were fewer than the invaded SACC-LM cells (Fig. 3A and B). The results were further confirmed by measurement of $\mathrm{OD}_{570}$ for the solution derived from the crystal violet discolored from the invaded cells by using $300 \mu \mathrm{l}$ of $33 \%$ glacial acetic acid $(0.207 \pm 0.055$ in SACC-83 vs. $0.717 \pm 0.001$ in SACC-LM; $\mathrm{P}<0.01$ ) (Fig. 3 C). These results indicate that SACC-LM has increased invasion ability compared to SACC-83.

Identification of molecular markers through $q P C R$ and western blotting. To identify the molecular markers of SACC- 83 and SACC-LM, we screened 136 genes which included 12 genes in the MMP family, 8 genes in Smads, 7 genes in BMPs, 13 genes in the S100 family, 17 genes in the cytokeratin family and 79 genes related to cancer cell proliferation and metastasis. With the exception of some genes that were expressed unstably in the two cell lines or showed no significant difference between SACC-83 and SACC-LM, 29 of the 136 genes showed significant differential expression in the two cell lines (Table I). Herein we presented 10 of 29 genes at the mRNA level, and 6 of 10 genes further confirmed by western blotting at the protein level. Real-time PCR results showed that compared with SACC-83, SACC-LM expressed MMP-3, MMP-9, MMP-14, S100A2, S100P, COX-2, EREG at high levels and MMP-1, MMP-2, MMP-13 at low levels (Fig. 4). Western blotting of MMP-1, MMP-2, MMP-9, MMP-14, COX-2, S100P further confirmed the qPCR results. In addition, western blotting results also showed that p-Akt, S100A4, MMP-12, MMP-17 were highly expressed in SACC-83 compared to SACC-LM (Fig. 5). 


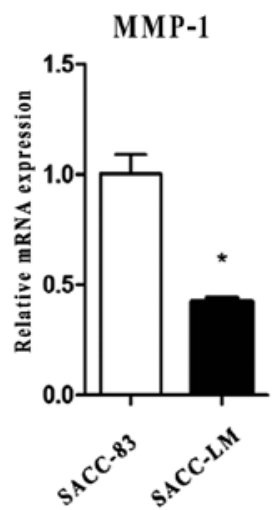

MMP-14

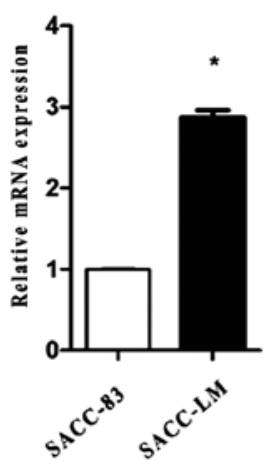

MMP-2

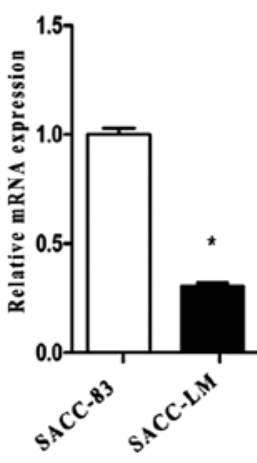

S100A2

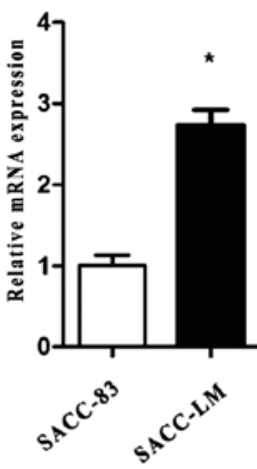

MMP-3

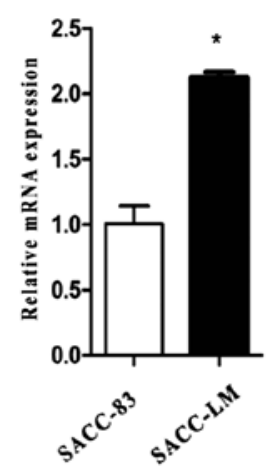

COX-2

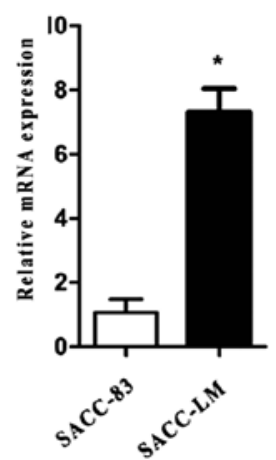

MMP-9

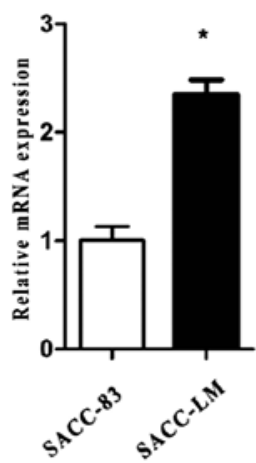

S100P

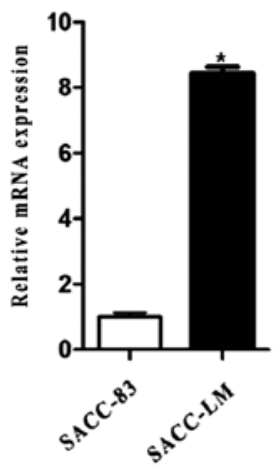

MMP-13

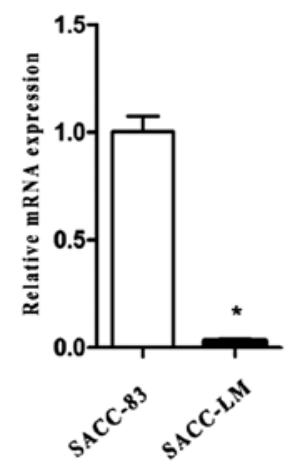

EREG

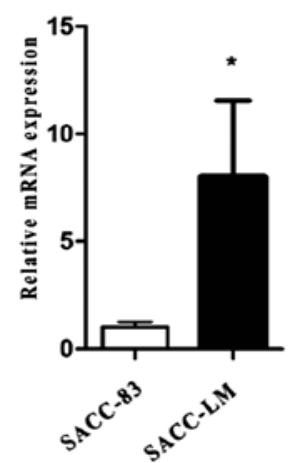

Figure 4. The expression profile of genes related in SACC-LM compared with SACC-83 by quantitative real-time PCR. "P<0.01.

\section{SACC-83 SACC-LM}

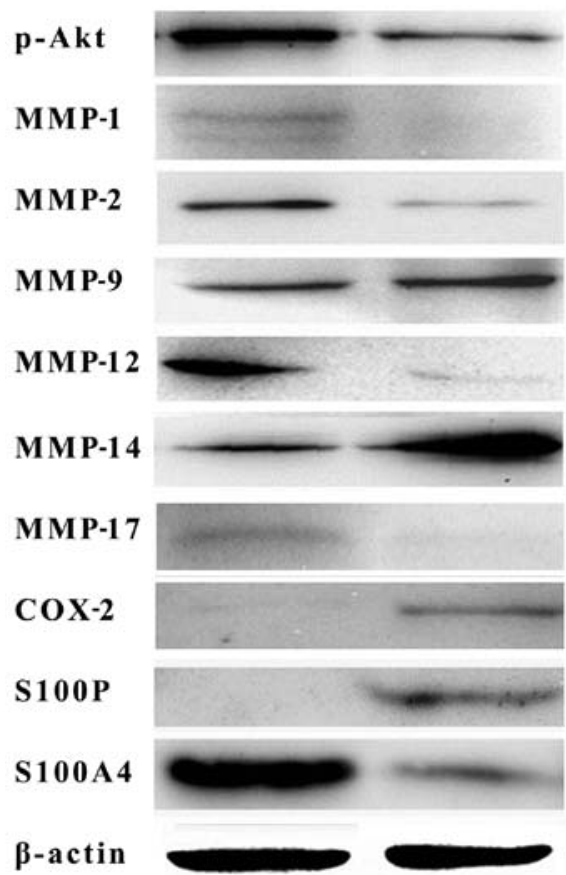

Figure 5. The differential expression genes at the protein level in SACC-83 and SACC-LM cells by western blotting.

\section{Discussion}

Cell lines are useful for a wide array of experiments in the life sciences. However, cell cross-contamination is increasing and requires further study (13-15). To date, false cell lines are widely distributed and used in the laboratories and cell banks worldwide. The German Cell Bank reported that nearly $20 \%$ of cell lines submitted to them were contaminated by different species or individuals (16). Several of the problems are related to the HeLa cell line which is cross-contaminated with 106 cell lines (3). Therefore, it is crucial and necessary that researchers know the cell lines they are studying and make scientific results reliable and reproducible.

A variety of methods are available for identifying crosscontamination including isozyme typing, HLA typing, karyotyping, DNA fingerprinting, short tandem repeat (STR). STR profiling is an inexpensive and reliable method to confirm cell line identity (17-20). In this study, SACC-83 and SACC-LM cells had identical STR profiling, which indicated that the SACC-83 and SACC-LM cell lines derived from the same patient. Compared with the HeLa STR profiling, SACC-83 and SACC-LM had $81.25 \%$ difference from HeLa in the 16 foci of STR profiling, indicating that SACC-83 and SACC-LM have as yet not been contaminated by HeLa cells.

Pan-cytokeratin and cytokeratin AE1 are the classical epithelial markers, which react specifically with a wide variety of normal and neoplastic epithelial tissues. Cytokeratin 8/18 and placental S100 (S100P) are the luminal markers which express in duct cells and duct carcinoma (21-24). SACC-83 and SACC-LM cells were positive for epithelial cells as well as for luminal marker CK8/18 and S100P. These indicated that both SACC-83 and SACC-LM originated from oral adenoepithelial cells.

However, it remains unclear whether SACC-83 and SACC-LM cross-contaminated each other. Owing to increasing 
research using SACC-83 and SACC-LM as models to explore the underlying mechanism of SACC, it is necessary to find the molecular features for identifying and avoiding cross-contamination and misuse of the two cell lines. Herein, we further investigated the invasion abilities of the two SACC cell lines. The transwell assay results showed the invasion ability of the cells was stable in these two cell lines. SACC-LM had increased invasion ability compared to SACC-83, which is consistent with previous studies (8). This indicated that SACC-83 and SACC-LM had different biological characteristics. It is well known that the gene determines the phenotype and the biological behavior of cells. Therefore, we next explored the expression of 136 genes at the mRNA level and examined the biomarkers to identify the two cell lines. Finally, 29 of 136 differential expression genes were identified via real-time PCR and western blotting.

Western blotting results showed that p-Akt expression is higher in SACC-83 than in SACC-LM, which further confirmed that SACC-LM cells were more vulnerable than SACC-83 under anoikis conditions (data not shown). In addition, MMP protein, particularly MMP-2 and MMP-9 are critical for cancer metastasis $(25,26)$. In general, concurrent overexpression of MMP-2 and MMP-9 contribute to invasion and metastasis of tumor cells by degrading extracellular matrix as well as promoting angiogenesis. Of note, compared with the parent cell line SACC-83, MMP-1, MMP-2, MMP-12 and MMP-13 were downregulated in the daughter cell line SACC-LM. However, other MMPs such as MMP-9, MMP-14 were upregulated in SACC-LM, which may contribute to the high invasion and lung metastasis ability. Other metastasisrelated genes including COX-2, S100P and EREG expression were higher in SACC-LM than in SACC-83, which indicated these genes could promote cancer spread. However, further investigations are required to elucidate the precise underlying mechanisms related to SACC metastasis.

In conclusion, SACC-83 and SACC-LM are homologous cancer cell lines, which are not contaminated by each other or other cancer cell lines. Compared with SACC-83, SACC-LM presents higher expression of COX-2, S100P, and lower expression of MMP-2, p-Akt, which could be the candidates for identifying the homologous cell lines SACC-83 and SACC-LM.

\section{Acknowledgements}

This study was supported by the National Natural Science Foundation of China (No. 81172556).

\section{References}

1. Lara-Padilla E and Caceres-Cortes JR: On the nature of the tumor-initiating cell. Curr Stem Cell Res Ther 7: 26-35, 2012.

2. Chatterjee R: Cell biology. Cases of mistaken identity. Science 315: 928-931, 2007.

3. Capes-Davis A, Theodosopoulos G, Atkin I, et al: Check your cultures! A list of cross-contaminated or misidentified cell lines. Int J Cancer 127: 1-8, 2010

4. Stacey GN: Cell contamination leads to inaccurate data: we must take action now. Nature 403: 356, 2000.
5. Parson W, Kirchebner R, Muhlmann R, et al: Cancer cell line identification by short tandem repeat profiling: power and limitations. FASEB J 19: 434-436, 2005.

6. Capes-Davis A, Reid YA, Kline MC, et al: Match criteria for human cell line authentication: Where do we draw the line? Int J Cancer 132: 2510-2519, 2013.

7. Li SL: Establishment of a human cancer cell line from adenoid cystic carcinoma of the minor salivary gland. Zhonghua Kou Qiang Yi Xue Za Zhi 25: 29-31, 62, 1990 (In Chinese).

8. Dong L, Wang YX, Li SL, et al: TGF-betal promotes migration and invasion of salivary adenoid cystic carcinoma. J Dent Res 90: 804-809, 2011.

9. Hu K, Li SL, Gan YH, Wang CY and Yu GY: Epiregulin promotes migration and invasion of salivary adenoid cystic carcinoma cell line SACC-83 through activation of ERK and Akt. Oral Oncol 45: 156-163, 2009.

10. Hu K, Gan YH, Li SL, et al: Relationship of activated extracellular signal-regulated kinase $1 / 2$ with lung metastasis in salivary adenoid cystic carcinoma. Oncol Rep 21: 137-143, 2009.

11. Kou XX, Hao T, Meng Z, Zhou YH and Gan YH: Acetylated Sp1 inhibits PTEN expression through binding to PTEN core promoter and recruitment of HDAC1 and promotes cancer cell migration and invasion. Carcinogenesis 34: 58-67, 2013.

12. Zhang JP and Li CY: The effect of transforming growth factorbetal on EDA region of fibronectin in oral squamous cell carcinoma and adenoid cystic carcinoma cells. Zhonghua Kou Qiang Yi Xue Za Zhi 42: 47-51, 2007 (In Chinese).

13. MacLeod RA, Dirks WG, Matsuo Y, Kaufmann M, Milch H and Drexler HG: Widespread intraspecies cross-contamination of human tumor cell lines arising at source. Int J Cancer 83: 555-563, 1999.

14. Lacroix M: Persistent use of 'false' cell lines. Int J Cancer 122: $1-4,2008$

15. Masters JR: Cell-line authentication: end the scandal of false cell lines. Nature 492: 186, 2012.

16. Masters J: False cell lines. Carcinogenesis 23: 371, 2002.

17. Masters JR: False cell lines: the problem and a solution. Cytotechnology 39: 69-74, 2002.

18. Azari S, Ahmadi N, Tehrani MJ and Shokri F: Profiling and authentication of human cell lines using short tandem repeat (STR) loci: Report from the National Cell Bank of Iran. Biologicals 35: 195-202, 2007.

19. American Type Culture Collection Standards Development Organization Workgroup ASN-0002: Cell line misidentification: the beginning of the end. Nat Rev Cancer 10: 441-448, 2010.

20. Barallon R, Bauer SR, Butler J, et al: Recommendation of short tandem repeat profiling for authenticating human cell lines, stem cells, and tissues. In Vitro Cell Dev Biol Anim 46: 727-732, 2010.

21. Shamloula MM, El-Shorbagy SH and Saied EM: P63 and cytokeratin8/18 expression in breast, atypical ductal hyperplasia, ductal carcinoma in situ and invasive duct carcinoma. J Egypt Natl Cancer Inst 19: 202-210, 2007.

22. Raspollini MR, Comin CE, Crisci A and Chilosi M: The use of placental S100 (S100P), GATA3 and napsin A in the differential diagnosis of primary adenocarcinoma of the bladder and bladder metastasis from adenocarcinoma of the lung. Pathologica 102: 33-35, 2010.

23. Esheba GE, Longacre TA, Atkins KA and Higgins JP: Expression of the urothelial differentiation markers GATA3 and placental $\mathrm{S} 100(\mathrm{~S} 100 \mathrm{P})$ in female genital tract transitional cell proliferations. Am J Surg Pathol 33: 347-353, 2009.

24. Saghravanian N, Mohtasham N and Jafarzadeh H: Comparison of immunohistochemical markers between adenoid cystic carcinoma and polymorphous low-grade adenocarcinoma. J Oral Sci 51: 509-514, 2009.

25. Philip S, Bulbule A and Kundu GC: Matrix metalloproteinase-2: mechanism and regulation of NF-kappaB-mediated activation and its role in cell motility and ECM-invasion. Glycoconj $\mathrm{J} 21$ : 429-441, 2004

26. Garavello W, Maggioni D, Nicolini G, Motta L, Tredici G and Gaini R: Association between metalloproteinases 2 and 9 activity and ERK1/2 phosphorylation status in head and neck cancers: An ex vivo study. Oncol Rep 24: 1073-1078, 2010. 